\title{
Low-Dimensional Dynamics of Populations of Pulse-Coupled Oscillators
}

\author{
Diego Pazó $^{1}$ and Ernest Montbrió ${ }^{2}$ \\ ${ }^{1}$ Instituto de Física de Cantabria (IFCA), CSIC-Universidad de Cantabria, 39005 Santander, Spain \\ ${ }^{2}$ Department of Information and Communication Technologies, Universitat Pompeu Fabra, \\ 08018 Barcelona, Spain
}

(Received 10 May 2013; revised manuscript received 10 December 2013; published 29 January 2014)

Large communities of biological oscillators show a prevalent tendency to self-organize in time. This cooperative phenomenon inspired Winfree to formulate a mathematical model that originated the theory of macroscopic synchronization. Despite its fundamental importance, a complete mathematical analysis of the model proposed by Winfree-consisting of a large population of all-to-all pulse-coupled oscillators-is still missing. Here, we show that the dynamics of the Winfree model evolves into the so-called OttAntonsen manifold. This important property allows for an exact description of this high-dimensional system in terms of a few macroscopic variables, and also allows for the full investigation of its dynamics. We find that brief pulses are capable of synchronizing heterogeneous ensembles that fail to synchronize with broad pulses, especially for certain phase-response curves. Finally, to further illustrate the potential of our results, we investigate the possibility of "chimera" states in populations of identical pulse-coupled oscillators. Chimeras are self-organized states in which the symmetry of a population is broken into a synchronous and an asynchronous part. Here, we derive three ordinary differential equations describing two coupled populations and uncover a variety of chimera states, including a new class with chaotic dynamics.

DOI: 10.1103/PhysRevX.4.011009

Subject Areas: Complex Systems, Nonlinear Dynamics

\section{INTRODUCTION}

In 1967, Winfree proposed the first mathematical model for the macroscopic synchronization observed in large populations of biological oscillators [1]. These natural systems typically achieve synchrony via brief pulselike signals emitted by the individual oscillators [2,3]. Wellknown examples of pulselike interactions are the action potentials emitted by neurons and other cells [4], the flashes of light emitted by fireflies [5], and the sound of hands in clapping audiences [6].

Assuming weak coupling, Winfree exploited the separation of time scales to characterize the state of each oscillator solely by its phase variable $\theta$. Using analytical arguments and numerical simulations, Winfree discovered that a population of $N \gg 1$ all-to-all-coupled phase oscillators showed a phase transition to macroscopic synchronization at a critical value of the "homogeneity" of the population [1,7]. Only a few years after Winfree's seminal paper, Kuramoto proposed a new phase model singularly amenable to mathematical analysis [8,9]. In an elegant and simple way, the Kuramoto model captures the transition to collective synchronization observed by Winfree and

Published by the American Physical Society under the terms of the Creative Commons Attribution 3.0 License. Further distribution of this work must maintain attribution to the author(s) and the published articles title, journal citation, and DOI. quickly became the canonical model to mathematically investigate synchronization phenomena [10].

The Kuramoto model has motivated a great deal of theoretical work and has been investigated under countless variations and used to model a number of physical, chemical, biological, social, and technological systems [3,11-13]. Yet, in 2008, Ott and Antonsen made a very important finding [14]: Kuramoto-like models have solutions in a reduced invariant manifold. This result drastically simplifies the task of investigating the collective dynamics of such systems.

However, despite their importance and generality, Kuramoto-like models-in which interactions are expressed by phase differences-are approximations of more-realistic models, such as the Winfree model, in the weak-coupling limit. Parameters of the original model do not usually have a simple mapping into the parameters of the Kuramoto-like model (see, e.g., Refs. $[15,16]$ ). In contrast to Kuramoto-like models, the Winfree model incorporates explicit pulselike interactions and phase response curves (PRCs) [17] that are customarily obtained from experiments [18] or from biologically realistic conductance-based models [19].

Thus far, theoretical attempts to understand the dynamics of the Winfree model have had very limited success. Beyond a valuable work in 2001 [20] and a few later studies [21], the lack of mathematical tractability of the model seems to be the drawback for its dissemination among scientists. 
In this paper we show that the Winfree model evolves into the so-called Ott-Antonsen (OA) manifold [14]. Under some circumstances-which we make clear below-this important property permits us to exactly describe this highdimensional system by two ordinary differential equations (ODEs). We exhaustively explore the effect of the PRC's shape and the pulse's width on the collective dynamics of the Winfree model. In general, the evolution of the Winfree model in the OA manifold opens the possibility of investigating phenomena that, thus far, have been addressed analytically using "Kuramoto oscillators." As an example, we uncover the existence of a variety of the so-called chimera states [22] in populations of "Winfree oscillators."

\section{WINFREE MODEL}

The Winfree model is written as

$$
\dot{\theta}_{i}=\omega_{i}+Q\left(\theta_{i}\right) \frac{\epsilon}{N} \sum_{j=1}^{N} P\left(\theta_{j}\right),
$$

where the overdot denotes the derivative with respect to time, the constant $\epsilon$ controls the coupling strength, and the oscillators are labeled by $i=1, \ldots ., N$. The presence of heterogeneity in the population is modeled via the natural frequencies $\omega_{i}$, which are drawn from a certain probability distribution $g(\omega)[1,20,21]$ (see also Ref. [23]). The PRC function $Q$ measures the degree of advance or delay of the phases when the oscillators are perturbed. Here, we adopt a PRC with a sinusoidal shape:

$$
Q(\theta)=\sigma-\sin (\theta+\beta) .
$$

A possible choice relating the offset $\sigma$ and the phase-lag parameter $\beta$ is $\sigma=\sin \beta$, so that the PRC vanishes at $\theta=0$, as is naturally assumed in neuronal modeling. If $\beta<\pi / 2$, neuronal oscillators are referred to as type-II, whereas $\beta=\pi / 2$ corresponds to a type-I neuronal oscillator [18,19,23-27].

We complete the definition of Eq. (1) with the smooth pulselike signal,

$$
P(\theta)=a_{n}(1+\cos \theta)^{n},
$$

where the integer parameter $n \geq 1$ allows us to control the width of the pulses. The normalizing constant $a_{n}$ is chosen so that the integral of $P(\theta)$ equals $2 \pi$. Thus, $a_{1}=1$, and for other values of $n, a_{n}=2^{n}(n !)^{2} /(2 n)$ !. Note also that the $n \rightarrow \infty$ limit of Eq. (3) is $P(\theta)=2 \pi \delta(\theta)$.

\section{LIMIT OF WEAK COUPLING AND NEARLY IDENTICAL FREQUENCIES}

We begin our analysis of the model defined by Eqs. (1-3) by taking the limit of small $\epsilon$ and the frequency diversity. Applying the classical perturbative averaging technique [9], we obtain

$$
\dot{\theta}_{i}^{(a v)}=\omega_{i}^{\prime}+\left(\frac{n}{n+1}\right) \frac{\epsilon}{N} \sum_{j=1}^{N} \sin \left[\theta_{j}^{(a v)}-\theta_{i}^{(a v)}-\beta\right],
$$

with $\omega_{i}^{\prime}=\omega_{i}+\epsilon \sigma$. Equation (4) is precisely the Kuramoto-Sakaguchi model [28]. An interesting outcome of our derivation of Eq. (4) is that, the narrower the pulses (the larger the $n$ values) in the original Winfree model, the stronger the effective coupling $\epsilon_{\text {eff }}=n \epsilon /(n+1)$.

In the case of a Lorentzian distribution of frequencies,

$$
g(\omega)=\frac{\Delta / \pi}{\left(\omega-\omega_{0}\right)^{2}+\Delta^{2}},
$$

a closed formula for the coupling at the emergence of a macroscopic cluster of synchronized oscillators exists [28]:

$$
\epsilon_{c}^{(a v)}=\frac{2 \Delta}{\cos \beta}\left(\frac{n+1}{n}\right) .
$$

Note that this linear dependence of $\epsilon_{c}$ on $\Delta$ is an approximation.

\section{LOW-DIMENSIONAL DYNAMICS OF THE WINFREE MODEL}

For the remainder of this paper, we analyze the Winfree model assuming neither weak coupling nor low frequency diversity. Our first key observation is that Eq. (1), with the PRC in Eq. (2), belongs to a family of models that can be written as

$$
\dot{\theta}_{i}(t)=\omega_{i}+B(t)+\operatorname{Im}\left[H(t) e^{-i \theta_{i}(t)}\right] .
$$

In our case, $B(t)=\epsilon \sigma h(t)$ and $H(t)=\epsilon e^{-i \beta} h(t)$, with the mean field

$$
h(t)=\frac{1}{N} \sum_{j=1}^{N} P\left(\theta_{j}(t)\right) .
$$

In the thermodynamic limit $N \rightarrow \infty$, systems of type (7) have solutions in the reduced invariant manifold discovered by Ott and Antonsen [14], which corresponds to a uniform distribution of certain constants of motion at each value of $\omega$ [29]. For $B=0$, it has been proven [30,31] that, provided the $\omega$ 's are drawn from a probability distribution function $g(\omega)$ that is differentiable and well-behaved in a certain way (see Ref. [31] for details), such as Lorentzian or Gaussian functions, the dynamics of Eq. (7) converges to the OA manifold. Remarkably, we have verified that the proof in Refs. [30,31] also holds for $B \neq 0$. Hence, next we apply the OA ansatz with the certainty that it captures the asymptotic dynamics of the model.

Let $F(\theta \mid \omega, t) d \theta$ be the fraction of oscillators with phases between $\theta$ and $\theta+d \theta$ and natural frequency $\omega$ at time $t$. The dynamics of $F$ is governed by the continuity equation 


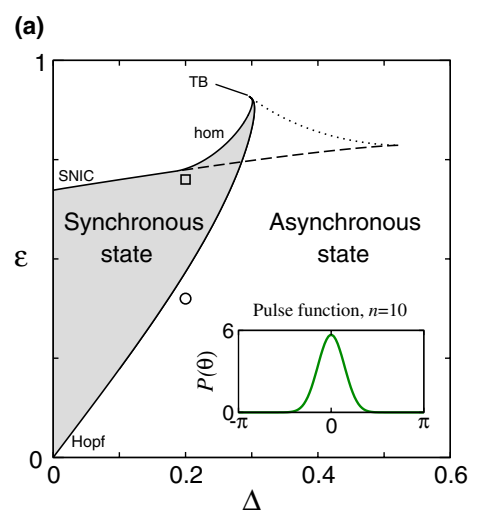

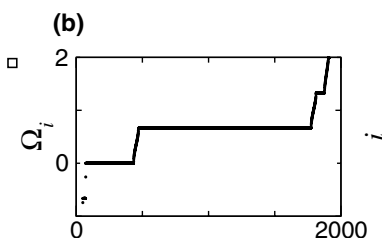
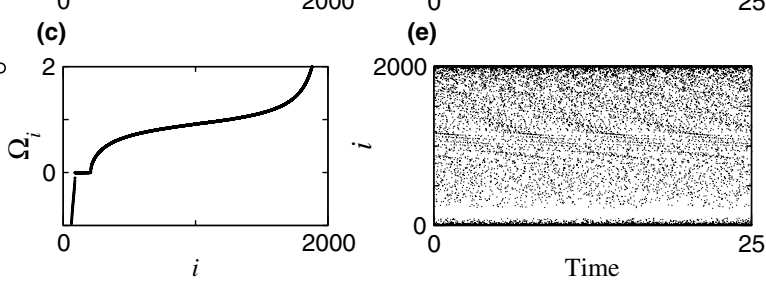

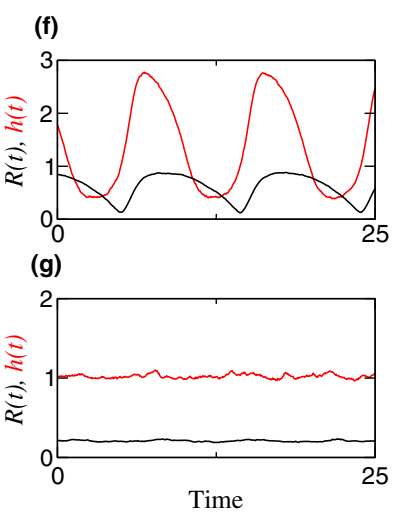

FIG. 1 (color online). (a) Phase diagram of model (1) obtained from the reduced Eq. (12), with $\beta=\sigma=0$ and $n=10$. Inset: Pulselike function [Eq. (3)]. Panels (b)-(g) show results obtained from the numerical integration of the Winfree model [Eq. (1)], with $N=2000$ oscillators and the natural frequencies selected deterministically to represent the Lorentzian distribution: $\omega_{i}=1+\Delta \tan [\pi / 2(2 i-N-1) /(N+1)]$, for $i=1, \ldots . ., N$. Two different points ( open square, open circle) corresponding to the synchronous (b),(d),(f), and asynchronous (c),(e),(g) states were chosen. (b),(c) Coupling-modified frequencies: $\Omega_{i}=$ $\lim _{t \rightarrow \infty} t^{-1} \int_{0}^{t} \dot{\theta}_{i}(t) d t$ versus the oscillators' index $i$. We observe that in the synchronization region, plateaus in $\Omega$ appear at a basic frequency and its integer multiples; other plateaus at rational multiples of the basic frequency are absent due to the purely sinusoidal form of the PRC; see Ref. [33]. (d),(e) Raster plots (points depicted whenever $\theta_{i}=0$ ). (f), (g) Time series of the modulus of the Kuramoto order parameter $R(t)=\left|N^{-1} \sum_{j} e^{i \theta_{j}}\right|$ and the mean field $h(t)$.

$\partial_{t} F=-\partial_{\theta}(\dot{\theta} F)$ since the number of oscillators is conserved. Using the OA ansatz,

$$
F(\theta \mid \omega, t)=\frac{1}{2 \pi}\left\{1+\left[\sum_{m=1}^{\infty} \alpha(\omega, t)^{m} e^{i m \theta}+\text { c.c. }\right]\right\}
$$

(where c.c. stands for complex conjugate), we find that $\alpha(\omega, t)$ necessarily obeys

$$
\partial_{t} \alpha=-i(\omega+B) \alpha+\frac{1}{2}\left(H^{*}-H \alpha^{2}\right) .
$$

This is still an infinite set of equations if the frequency distribution is continuous. Fortunately, a drastic simplification is possible if $g(\omega)$ has a finite number of simple poles off the real axis-as it does for the Lorentzian distribution (5); see below.

For the analysis that follows, it is convenient to use the generalized order parameters [32]

$$
Z_{m}(t)=\int_{-\infty}^{\infty} g(\omega) \int_{0}^{2 \pi} F(\theta \mid \omega, t) e^{i m \theta} d \theta d \omega,
$$

with $m \in \mathbb{N}$. Recalling the ansatz [Eq. (9)], and noting that $\alpha$ admits an analytical continuation into the lower-half complex $\omega$ plane [14], we can evaluate Eq. (11) by applying the residue theorem. Since the Lorentzian function [Eq. (5)] has one simple pole $\omega^{p}=\omega_{0}-i \Delta$ inside the contour, we find that all order parameters depend on the value of $\alpha$ at the pole $Z_{m}(t)=\left[\alpha\left(\omega^{p}, t\right)^{*}\right]^{m}$. The dynamics of the Kuramoto order parameter $Z_{1} \equiv R e^{i \Psi}$ is governed by two ODEs obtained equating $\omega=\omega^{p}$ in Eq. (10):

$$
\begin{array}{r}
\dot{R}=-\Delta R+\frac{\epsilon h}{2}\left(1-R^{2}\right) \cos (\Psi+\beta), \\
\dot{\Psi}=\omega_{0}+\epsilon h\left[\sigma-\frac{1+R^{2}}{2 R} \sin (\Psi+\beta)\right] .
\end{array}
$$

Remarkably, these two ODEs describe exactly the Winfree model dynamics, irrespective of the particular interaction function $P(\theta)$. In order to close Eq. (12), we consider $P(\theta)$ to be the pulselike function in Eq. (3) and express the mean field Eq. (8) in terms of $R$ and $\Psi$. For $n=1$, the result is trivial: $h_{1}=1+R \cos \Psi$. For $n>1$, with the important observation that the generalized order parameters are powers of the Kuramoto order parameter $Z_{m}=Z_{1}^{m}$ [29], after some algebra we obtain

$$
h_{n}(R, \Psi)=1+2(n !)^{2} \sum_{k=1}^{n} \frac{R^{k} \cos (k \Psi)}{(n+k) !(n-k) !} .
$$

Equation (12) cannot be solved analytically, but the loci of the bifurcations, where the qualitative behavior changes, can easily be found by standard numerical continuation techniques. We rescale $\Delta, \epsilon$, and time by $\omega_{0}$, so that $\omega_{0}=1$ hereafter. Additionally, we select $\sigma=\sin \beta$-see the insets in Fig. 2. We observe that the results are qualitatively the same independently of $n$ and $\beta$; hence, the phase diagram for $\beta=0$ and $n=10$ in Fig. 1(a) accounts for all of the phenomenology of the model.

The case $\beta=0$ was already studied in Ref. [20] for a uniform distribution $g(\omega)$ obtaining a similar result, albeit some differences show up due to the different support of the distributions. In the phase diagram of Fig. 1(a), a Hopf 
bifurcation line emanates from the origin-with the slope predicted by Eq. (6) - limiting the shaded region of synchronization together with the other solid lines. In the synchronous state, a macroscopic cluster of oscillators rotates with the same coupling-modified frequency $\Omega$, and as a result, the order parameter and the mean field oscillate; see Figs. 1(b) and 1(f). Note that, in addition, a cluster of oscillators with $\Omega=0$ and quivering near $\theta=0$ is present for all $\epsilon>0$. The region of synchronization is bounded at large values of $\epsilon$ by a homoclinic (hom) and a saddle node on the invariant cycle (SNIC) bifurcations. The latter bifurcation line intercepts the $\epsilon$ axis at $(n+1)^{n+1} /\left[a_{n}(2 n+1)^{n+1 / 2}\right]$, i.e., $\epsilon=0.6735 \ldots$. , for $n=10$. In the phase diagram of Fig. 1(a), we see that the Hopf line ends at a Takens-Bogdanov (TB) point [34], which with two other (codimension-two) points organizes the region where Hopf and SNIC bifurcations meet; this conveys bistability between the synchronous and the asynchronous states inside a small region bounded by the dashed (saddle-node bifurcation), Hopf, and homoclinic lines.

After the preliminary introduction to the model dynamics, we focus on the effect that the pulses' shape and the oscillators' PRC has on the phase diagram of Fig. 1(a). The boundaries in Fig. 2(a) for $n=1$ and 10 show that the region of synchronization enlarges as $n$ grows, as suggested by Eq. (6). It is interesting to note that the coordinate $\epsilon$ of the TB point diverges with $n$, while the $\epsilon$ values of the SNIC line decrease. As a result, the region of bistability widens as $n$ grows since the SNIC bifurcation at the $\epsilon$ axis approaches the finite value $\sqrt{\frac{e}{2 \pi}}=0.6577 \ldots$ as $n \rightarrow \infty$, while the $\epsilon$ coordinate of the TB point progressively grows. The study of large $n$ values is difficult due to the highly convoluted form of Eq. (13). It is, therefore, useful from a mathematical perspective to consider the idealization $P(\theta)=2 \pi \delta(\theta)$. Using the trigonometric representation of the Dirac delta function, we obtain the mean field:
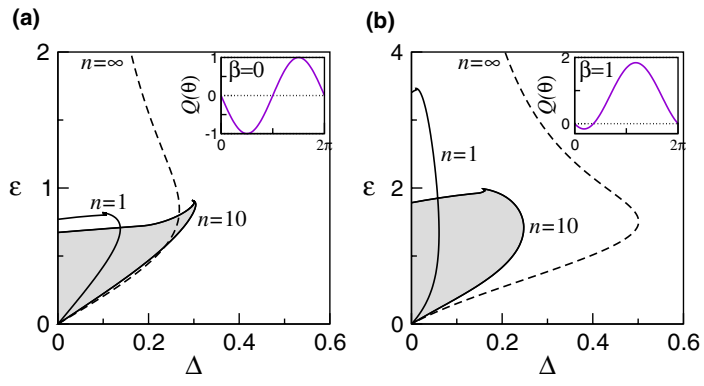

FIG. 2 (color online). Synchronization boundaries for PRCs with $\sigma=\sin \beta$, where (a) $\beta=0$ and (b) $\beta=1$, and for pulselike interactions (3) with $n=1,10$, and $\infty$ (Dirac's delta). Insets: PRCs $Q(\theta)$, see Eq. (2). The region of synchronization for $n=$ 10 appears shaded.

$$
h_{\infty}(R, \Psi)=\frac{1-R^{2}}{1-2 R \cos \Psi+R^{2}} .
$$

In this derivation, the $n \rightarrow \infty$ limit is taken after the $N \rightarrow$ $\infty$ limit, and, therefore, any subsequent result using $h_{\infty}$ is expected to be a truly asymptotic one as $n$ grows provided $N$ is kept sufficiently large. On the contrary, implementing instantaneous interactions $(n=\infty)$ with a finite population $(N<\infty)$ cannot fit in the theory since the mentioned limits do not commute (this noncommutativity was studied in Ref. [35] for a model of leaky integrate-and-fire neurons).

Inserting $h_{\infty}$ in Eq. (12), we obtain the boundaries [36] shown with dashed lines in Figs. 2(a) and 2(b). We see that, for $\beta=0$, there is already a noticeable similarity between the regions of synchronization for $n=10$ and $n=\infty$. The main discrepancy is observed at high $\epsilon$ values, which is not particularly interesting since, in any case, almost the whole population does not rotate in that region (see Ref. [37] for a description of this effect). As stated above, as $n$ grows, the TB point moves upwards, so that the synchronization regions eventually match at the $n \rightarrow \infty$ limit (note, nevertheless, that the limit is somewhat singular because the bistability region disappears).

For $\beta=1$, see Fig. 2(b), the difference between the results for $n=10$ and $n=\infty$ becomes apparent, and more tangible than what could be naively expected from Eq. (6). In fact, the closer $\beta$ approaches to $\pi / 2$, the more favorable is a sharp $P(\theta)$ to achieve synchronization. We claim this is a general statement, since we have also observed it numerically with Gaussian $g(\omega)$. It may be conjectured that the effectiveness of sharp spikes to achieve synchronization is one reason for their ubiquity in nature.

\section{TWO COUPLED POPULATIONS: CHIMERA STATES}

Finally, we illustrate how our results permit us to investigate problems that, thus far, were addressed only analytically using the Kuramoto model. Recently, an interesting dynamical state, called chimera, has been discovered in which identical oscillators with identical connectivity self-organize into clusters with different synchronous behavior [22]. In this state, complete synchronization of all of the oscillators is a stable solution and, therefore, the chimera state does not appear via a usual symmetry-breaking mechanism [38]. The simplest setup capable of sustaining chimeras, in both experimental $[39,40]$ and numerical $[41,42]$ realizations, consists of two coupled subpopulations $b=(1,2)$ of identical oscillators. Here, we consider a pulselike coupling with the positive constants $\mu$ and $\nu$ controlling intrapopulation and interpopulation interactions, respectively,

$$
\dot{\theta}_{i}^{(b)}=1+Q\left(\theta_{i}^{(b)}\right)\left[\frac{\mu}{N_{b}} \sum_{j=1}^{N_{b}} P\left(\theta_{j}^{(b)}\right)+\frac{\nu}{N_{b^{\prime}}} \sum_{j=1}^{N_{b^{\prime}}} P\left(\theta_{j}^{\left(b^{\prime}\right)}\right)\right],
$$


with $b^{\prime}=(2,1)$. Note that the equation for each subpopulation has the structure of Eq. (7), with $\omega_{i}=1$, $B \sigma^{-1}=H e^{i \beta}=\mu h^{(b)}+\nu h^{\left(b^{\prime}\right)}$, and $h^{(b)}=\frac{1}{N_{b}} \sum_{j=1}^{N_{b}} P\left(\theta_{j}^{(b)}\right)$. Consequently, there is a solution in which each subsystem evolves into its own OA manifold (9). The absence of diversity in the populations makes the OA manifold neutrally stable $[29,43,44]$. Nevertheless, the OA manifold becomes attracting as soon as a tiny amount of diversity is present [45]. Thus, in some sense, the OA manifold is the "skeleton" of the phase space, and it is legitimate to analyze the system with the OA ansatz.

The ODEs governing the dynamics of the order parameter of the $b$ th subpopulation $Z_{1}^{(b)} \equiv R_{b} e^{i \Psi_{b}}-$ cf. Eq. (12)—are

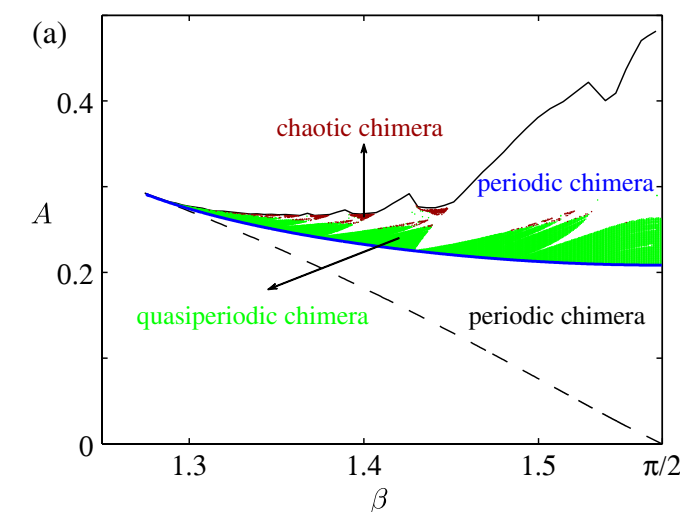

(b)

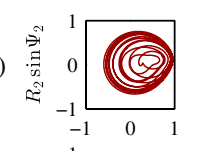

(c)

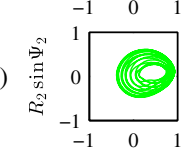

(d)

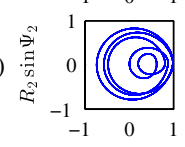

(e)
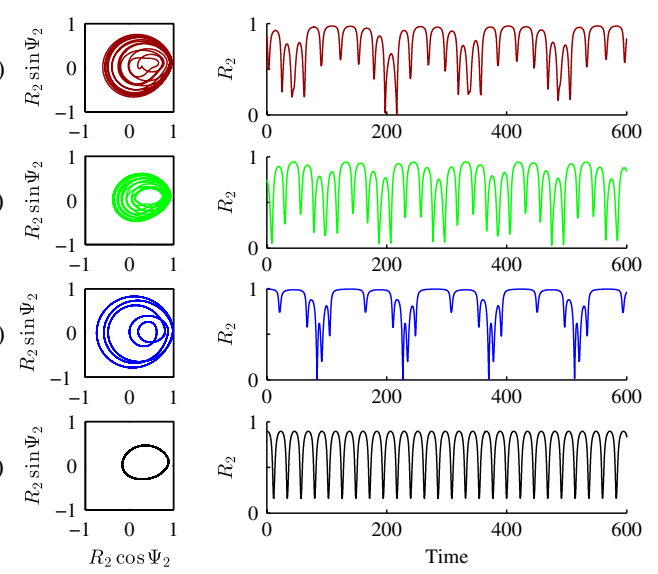

FIG. 3 (color online). (a) Location of different chimera types in the $(\beta, A)$ plane for $\sigma=0, n=1$, and $S=0.5$ (similar results are obtained in a wide range of $\sigma, S$, and $n$ ). Chimeras exist between the dashed line (the locus of a saddle-node bifurcation of limit cycles) and the solid line (corresponding to both the boundary crisis $[46,47]$ and the saddle-node bifurcation of cycles). Above the thick (blue) line, quasiperiodic and chaotic chimeras are found in the light (green) and dark (red) shaded regions, respectively. (b)-(e) Trajectories projected onto $R_{2} e^{i \Psi_{2}}$ and $R_{2}(t)$ for the parameter values with distinct behaviors: $(\beta, A)=(1.4,0.265),(1.42,0.24),(1.5,0.35)$, and $(1.45,0.19)$ from (b) to (e), corresponding to chaotic, quasiperiodic, and periodic chimera states above and below the thick (blue) line, respectively.

$$
\begin{aligned}
& \dot{R}_{b}=\frac{\mu h^{(b)}+\nu h^{\left(b^{\prime}\right)}}{2}\left(1-R_{b}^{2}\right) \cos \left(\Psi_{b}+\beta\right), \\
& \dot{\Psi}_{b}=1+\left[\mu h^{(b)}+\nu h^{\left(b^{\prime}\right)}\right]\left[\sigma-\frac{1+R_{b}^{2}}{2 R_{b}} \sin \left(\Psi_{b}+\beta\right)\right] .
\end{aligned}
$$

Because we are interested in states where one subpopulation is fully synchronized, say, the first one $\left(R_{1}=1\right)$, the equation for $R_{1}$ disappears. We then obtain a system of only three ODEs (for $\Psi_{1}, R_{2}$, and $\Psi_{2}$ ) that makes it possible to carry out an exhaustive exploration of the chimera states.

It is convenient for the analysis to define two parameters: $A=(\mu-\nu) /(\mu+\nu)$, quantifying the imbalance between intrapopulation and interpopulation interactions, and $S=\mu+\nu$, quantifying the coupling strength. Interestingly, in the limit of $\mu, \nu \rightarrow 0$, irrespective of the values of $\sigma$ and $n$, the system reduces (via averaging) to two ODEs for $R_{2}$ and $\psi=\Psi_{1}-\Psi_{2}$ identical to those in Eq. (12) of Abrams et al. [41] for oscillators of the Kuramoto-Sakaguchi type. Hence, for $S \rightarrow 0$, we can borrow the results in Ref. [41], in particular, the existence of chimeras only for $\beta$ values not far from $\pi / 2$. However, if $S$ is not small, the system behaves as genuinely three dimensional. The structure of the phase diagram in Fig. 3(a) is reminiscent of the one in Fig. 4 of Ref. [41], but now a much richer scenario emerges due to the additional degree of freedom. Above the Neimark-Sacker (or secondary Hopf) bifurcation, signaled by a thick (blue) line, we find quasiperiodic chimeras and the expected resonance tongues corresponding to limit cycles on the surface of the invariant torus. As we move away from the Neimark-Sacker bifurcation, the torus breaks down [46] and the resonances merge, giving rise to an intricate set of bifurcations (not shown, see Ref. [48]). Perhaps the most remarkable consequence of the torus breakdown is the existence of chaotic chimera states in the dark (red) shaded region of the phase diagram in Fig. 3(a). Figures 3(b)-3(e) show trajectories projected onto the $R_{2} e^{i \Psi_{2}}$ plane and time series $R_{2}(t)$ for specific values of $\beta$ and $A$.

\section{CONCLUSIONS}

The Winfree model describes a population of heterogeneous limit cycle oscillators, which interact via pulselike signals. Our most important finding is that the Winfree model with sinusoidal PRC, see Eq. (2), belongs to a family of systems with the form of Eq. (7), and that such systems have asymptotic dynamics in a reduced space, called the Ott-Antonsen manifold. This important property allows us to exactly describe the dynamics of the Winfree model with only two ODEs, Eq. (12), in the case of Lorentzian frequency distribution. The phase diagrams in Figs. 1 and 2 permit us to understand the effect of four parameters, $\Delta, \epsilon, \beta$, and $n$, controlling the spread of the natural frequencies, the coupling strength, the PRC, and the pulses' width, respectively. Interestingly, we find that brief pulses 
(large $n$ values) are capable of synchronizing heterogeneous ensembles that fail to synchronize with broad pulses. This feature of brief pulses is increasingly enhanced as the PRC becomes more off centered (increasing $\beta$ ), i.e., as it approaches type-I PRCs; see Fig. 2(b). It is worth noting that this property is not captured by applying averaging, see Eq. (6), since the approximation [Eq. (4)] holds only at low values of coupling and frequency heterogeneity. Finally, the potential of our findings is illustrated by uncovering a variety of chimera states in networks of pulse-coupled oscillators, which include a new class of chimeras with chaotic dynamics.

Our work suggests a number of future lines of research. For example, it would be interesting to investigate the dynamics of the Winfree model with more-realistic ingredients, such as time-delayed interactions or pulselike functions with coupling kinetics. In addition, our theory can readily incorporate external fields and multimodal frequency distributions. All in all, we believe our results will foster theoretical advances on the collective dynamics of oscillators' systems, upgrading the mathematical basis of macroscopic synchronization beyond Kuramoto-like models.

\section{ACKNOWLEDGMENTS}

We thank Juan M. López for a critical reading of the manuscript, Arkady Pikovsky for interesting discussions, and John Rinzel for pointing us to Ref. [7]. D. P. acknowledges support from Cantabria International Campus and the Ramón y Cajal program of MINECO (Spain). We acknowledge support from the Spanish research Projects No. FIS2009-12964-C05-05 and No. SAF2010-16085.

Note added.-Recently, it came to our attention that, in parallel to our work, other authors have used the OA ansatz to study ensembles of pulse-coupled theta neurons [49].

[1] A. T. Winfree, Biological Rhythms and the Behavior of Populations of Coupled Oscillators, J. Theor. Biol. 16, 15 (1967).

[2] A. T. Winfree, The Geometry of Biological Time (Springer, New York, 1980).

[3] S. H. Strogatz, Sync: The Emerging Science of Spontaneous Order (Hyperion Press, New York, 2003).

[4] A. L. Hodgkin and A. F. Huxley, A Quantitative Description of Membrane Current and Its Application to Conduction and Excitation in Nerve, J. Physiol. 117, 500 (1952).

[5] J. Buck and E. Buck, Mechanism of Rhythmic Synchronous Flashing of Fireflies, Science 159, 1319 (1968).

[6] Z. Néda, E. Ravasz, Y. Brechet, T. Vicsek, and A.-L. Barabási, Self-Organizing Processes: The Sound of Many Hands Clapping, Nature (London) 403, 849 (2000).

[7] A. T. Winfree, in Nonlinear Oscillations in Biology, Lectures in Applied Mathematics Vol. 17, F. C. Hoppensteadt (American Mathematical Society, Providence, 1979) pp. 93-126.
[8] Y. Kuramoto, in International Symposium on Mathematical Problems in Theoretical Physics, Lecture Notes in Physics Vol. 39, edited by H. Araki (Springer, Berlin, 1975) pp. 420-422.

[9] Y. Kuramoto, Chemical Oscillations, Waves, and Turbulence (Springer-Verlag, Berlin, 1984).

[10] S. H. Strogatz, From Kuramoto to Crawford: Exploring the Onset of Synchronization in Populations of Coupled Oscillators, Physica (Amsterdam) 143D, 1 (2000).

[11] A. S. Pikovsky, M. G. Rosenblum, and J. Kurths, Synchronization, A Universal Concept in Nonlinear Sciences (Cambridge University Press, Cambridge, England, 2001).

[12] S. C. Manrubia, S. S. Mikhailov, and D. H. Zanette, Emergence of Dynamical Order (World Scientific, Singapore, 2004).

[13] J. A. Acebrón, L. L. Bonilla, C. J. Pérez Vicente, F. Ritort, and R. Spigler, The Kuramoto Model: A Simple Paradigm for Synchronization Phenomena, Rev. Mod. Phys. 77, 137 (2005).

[14] E. Ott and T. M. Antonsen, Low-Dimensional Behavior of Large Systems of Globally Coupled Oscillators, Chaos 18, 037113 (2008).

[15] K. Wiesenfeld, P. Colet, and S. H. Strogatz, Synchronization Transitions in a Disordered Josephson Series Array, Phys. Rev. Lett. 76, 404 (1996).

[16] E. Montbrió and D. Pazó, Collective Synchronization in the Presence of Reactive Coupling and Shear Diversity, Phys. Rev. E 84, 046206 (2011).

[17] C. C. Canavier, Phase Response Curve, Scholarpedia 1, 1332 (2006); Phase Response Curves in Neuroscience, edited by N. W. Schultheiss, A. A. Prinz, and R. J. Butera (Springer, New York, 2012).

[18] T. Tateno and H.P.C. Robinson, Phase Resetting Curves and Oscillatory Stability in Interneurons of Rat Somatosensory Cortex, Biophys. J. 92, 683 (2007); B. Kralemann, M. Frühwirth, A. Pikovsky, M. Rosenblum, T. Kenner, J. Schaefer, and M. Moser, In Vivo Cardiac Phase Response Curve Elucidates Human Respiratory Heart Rate Variability, Nat. Commun. 4, 2418 (2013).

[19] E. M. Izhikevich, Dynamical Systems in Neuroscience (MIT Press, Cambridge, MA, 2007).

[20] J. T. Ariaratnam and S. H. Strogatz, Phase Diagram for the Winfree Model of Coupled Nonlinear Oscillators, Phys. Rev. Lett. 86, 4278 (2001).

[21] D. D. Quinn, R. H. Rand, and S. H. Strogatz, Singular Unlocking Transition in the Winfree Model of Coupled Oscillators, Phys. Rev. E 75, 036218 (2007); L. Basnarkov and V. Urumov, Critical Exponents of the Transition from Incoherence to Partial Oscillation Death in the Winfree Model, J. Stat. Mech. (2009) P10014.

[22] A. E. Motter, Nonlinear Dynamics: Spontaneous Synchrony Breaking, Nat. Phys. 6, 164 (2010).

[23] Y. Tsubo, J. N. Teramae, and T. Fukai, Synchronization of Excitatory Neurons with Strongly Heterogeneous Phase Responses, Phys. Rev. Lett. 99, 228101 (2007).

[24] D. Hansel, G. Mato, and C. Meunier, Synchrony in Excitatory Neural Networks, Neural Comput. 7, 307 (1995).

[25] B. Ermentrout, Type I Membranes, Phase Resetting Curves, and Synchrony, Neural Comput. 8, 979 (1996). 
[26] L. Neltner, D. Hansel, G. Mato, and C. Meunier, Synchrony in Heterogeneous Networks of Spiking Neurons, Neural Comput. 12, 1607 (2000).

[27] P. Goel and B. Ermentrout, Synchrony, Stability, and Firing Patterns in Pulse-Coupled Oscillators, Physica (Amsterdam) 163D, 191 (2002).

[28] H. Sakaguchi and Y. Kuramoto, A Soluble Active Rotator Model Showing Phase Transitions via Mutual Entrainment, Prog. Theor. Phys. 76, 576 (1986); O. E. Omel'chenko and M. Wolfrum, Nonuniversal Transitions to Synchrony in the Sakaguchi-Kuramoto Model, Phys. Rev. Lett. 109, 164101 (2012).

[29] A. Pikovsky and M. Rosenblum, Dynamics of Heterogeneous Oscillator Ensembles in Terms of Collective Variables, Physica (Amsterdam) 240D, 872 (2011).

[30] E. Ott and T. M. Antonsen, Long-Time Evolution of Phase Oscillator Systems, Chaos 19, 023117 (2009).

[31] E. Ott, B. R. Hunt, and T. M. Antonsen, Comment on "Long-Time Evolution of Phase Oscillator Systems", Chaos 21, 025112 (2011).

[32] H. Daido, Onset of Cooperative Entrainment in Limit-Cycle Oscillators with Uniform All-to-All Interactions: Bifurcation of the Order Function, Physica (Amsterdam) 91D, 24 (1996).

[33] J. R. Engelbrecht and R. Mirollo, Structure of Long-Term Average Frequencies for Kuramoto Oscillator Systems, Phys. Rev. Lett. 109, 034103 (2012).

[34] Y. A. Kuznetsov, Elements of Applied Bifurcation Theory (Springer-Verlag, New York, 1998).

[35] R. Zillmer, R. Livi, A. Politi, and A. Torcini, Stability of the Splay State in Pulse-Coupled Networks, Phys. Rev. E 76, 046102 (2007).

[36] For $\beta=0$, the boundary is at $\epsilon_{c}=\left(1+5 \Delta^{2} \pm\right.$ $\left.\sqrt{1-14 \Delta^{2}+\Delta^{4}}\right) / 6 \Delta$ with $\Delta \leq 2-\sqrt{3}$.

[37] B. Ermentrout and N. Kopell, Oscillator Death in Systems of Coupled Neural Oscillators, SIAM J. Appl. Math. 50, 125 (1990).

[38] Note that this is not the case of the chimeralike state found in ensembles of leaky integrate-and-fire oscillators in S. Olmi, A. Politi, and A. Torcini, Collective Chaos in Pulse-Coupled Neural Networks, Europhys. Lett. 92, 60007 (2010).

[39] M. R. Tinsley, S. Nkomo, and K. Showalter, Chimera and Phase-Cluster States in Populations of Coupled Chemical Oscillators, Nat. Phys. 8, 662 (2012).

[40] E. A. Martens, S. Thutupalli, A. Fourrière, and O. Hallatschek, Chimera States in Mechanical Oscillator Networks, Proc. Natl. Acad. Sci. U.S.A. 110, 10563 (2013).

[41] D. M. Abrams, R. Mirollo, S. H. Strogatz, and D. A. Wiley, Solvable Model for Chimera States of Coupled Oscillators, Phys. Rev. Lett. 101, 084103 (2008).

[42] E. Montbrió, J. Kurths, and B. Blasius, Synchronization of Two Interacting Populations of Oscillators, Phys. Rev. E 70, 056125 (2004).

[43] A. Pikovsky and M. Rosenblum, Partially Integrable Dynamics of Hierarchical Populations of Coupled Oscillators, Phys. Rev. Lett. 101, 264103 (2008).

[44] S. Watanabe and S. H. Strogatz, Constant of Motion for Superconducting Josephson Arrays, Physica (Amsterdam) 74D, 197 (1994).

[45] C. R. Laing, Chimera States in Heterogeneous Networks, Chaos 19, 013113 (2009).

[46] V. Afraimovich, V. Arnol'd, Y. Il'yashenko, and L. Shil'nikov, in Dynamical Systems, V, Encyclopaedia of Mathematical Sciences, edited by V. Arnol'd (SpringerVerlag, Berlin, 1994).

[47] E. Ott, Chaos in Dynamical Systems (Cambridge University Press, Cambridge, England, 2002).

[48] V. Kirk, Merging of Resonance Tongues, Physica (Amsterdam) 66D, 267 (1993).

[49] P. So, T. B. Luke, and E. Barreto, Networks of Theta Neurons with Time-Varying Excitability: Macroscopic Chaos, Multistability, and Final-State Uncertainty, Physica (Amsterdam) 267D, 16 (2014); T. B. Luke, E. Barreto, and P. So, Complete Classification of the Macroscopic Behavior of a Heterogeneous Network of Theta Neurons, Neural Comput. 25, 3207 (2013). 